\title{
New Periodic Wave Solutions for MNW Hierarchy with the Aid of Computerized Symbolic Computation
}

\author{
Sheng Zhang ${ }^{1,}$, Luyao Zhang ${ }^{1, b}$ \\ ${ }^{1}$ School of Mathematics and Physics, Bohai University, Jinzhou, 121013, China \\ aemail: szhangchina@126.com, bemail: 348564207@163.com
}

Keywords: Periodic wave solution; Jacobi elliptic function solution; Solitary wave solution; Trigonometric function solution; F-expansion method; MNW hierarchy

\begin{abstract}
Computer has received more and more applications in various fields. In this paper, many new periodic wave solutions expressed by Jacobi elliptic functions of the Mikhauilov-NovikovWang (MNW) hierarchy are obtained by using the extended F-expansion method. In the limit cases, the obtained Jacobi elliptic function solutions degenerate into solitary wave solutions and trigonometric function solutions. It is shown that the extended F-expansion method with the aid of computerized symblic computation can provide a preferred mathematical tool for finding new periodic wave solutions of nonlinear partial differential equations (PDEs).
\end{abstract}

\section{Introduction}

In a recent work [1], starting from the stationaly zero-curvature equation associated with a $3 \times 3$ matrix spectral problem Shan and Zhu derived a new hierarchy of nonlinear evolution equations (NLEEs) called the Mikhauilov-Novikov-Wang (MNW) hierarchy which includes two cases

$\left.u_{t}=-\left(d_{2 m+1, x}+h_{2 m+1}\right), \quad v_{t}=\left(v a_{2 m}\right)_{x}-2 v b_{2 m}\right), \quad m \geq 0$,

and

$\left.u_{t}=-\left(d_{2 m+2, x}+h_{2 m+2}\right), \quad v_{t}=\left(v a_{2 m+1}\right)_{x}-2 v b_{2 m+1}\right), \quad m \geq 0$,

by using the Lie algebra approach. In Eqs. (1) and (2), the coefficient functions $a_{2 m}, a_{2 m+1}, b_{2 m}$, $b_{2 m+1}, d_{2 m+1}, d_{2 m+2}, h_{2 m+1}$, and $h_{2 m+2}$ are determined by the following recursive relations

$a_{m x}=-b_{m}, \quad b_{m x}=e_{m}-u a_{m}-d_{m+1}-v d_{m-1}, \quad c_{m x}=u h_{m}-f_{m}, d_{m x}=2 h_{m}, \quad e_{m x}=u b_{m}-h_{m+1}-v h_{m-1}$, (3)

$f_{m x}=-\left(3 u c_{m}+g_{m}\right)+a_{m+1}+v a_{m-1}, \quad g_{m x}=-2 u f_{m}+2 b_{m+1}+2 v b_{m-1}, \quad h_{m x}=3 c_{m}+u d_{m}, \quad m \geq 1$,

$b_{0 x}=e_{0}-u a_{0}-d_{1}, e_{0 x}=u b_{0}-h_{1}, f_{0 x}=-\left(3 u c_{0}+g_{0}\right)+a_{1}, g_{0 x}=-2 u f_{0}+2 b_{1}$,

with the different initial values

$a_{0}=b_{0}=c_{0}=d_{0}=f_{0}=h_{0}=g_{0}=0, e_{0}=\alpha,(\alpha=$ const., $\alpha \neq 0)$,

and

$a_{0}=b_{0}=c_{0}=d_{0}=f_{0}=h_{0}=e_{0}=0, g_{0}=\beta,(\alpha=$ const., $\beta \neq 0)$,

For the initial values (6), when $m=1$ and $\alpha=9$, Eq. (1) reduces to the MNW equations

$u_{t}=-u_{x x x x x}+10 u u_{x x x}+25 u_{x} u_{x x}-20 u_{x} u_{x x}+9 v_{x}, \quad v_{t}=3 v u_{x x x}-4 v_{x} u^{2}+v_{x} u_{x x}-24 v u u_{x}$,

which can be converted into the well-known Kaup-Kupershmidt equation

$u_{t}=-u_{x x x x x}+10 u u_{x x x}+25 u_{x} u_{x x}-20 u_{x} u_{x x}$,

as long as setting $v=0$.

For the initial values (7), when $m=1$ and $\beta=27$, Eq. (2) gives a new system of NLEEs

$$
\begin{aligned}
u_{t}= & 18 v_{x x x}-36(v u)_{x}-u_{7 x}+49 u_{x} u_{x x x x}+14 u u_{5 x}+84 u_{x x} u_{x x x}-70 u_{x}^{3} \\
& -252 u u_{x} u_{x x}-56 u^{2} u_{x x x}+\frac{224}{3} u^{3} u_{x}, \\
v_{t}= & -36 v v_{x}+v_{x} v_{x x x x}+3 v u_{5 x}-12 v_{x} u u_{x x}-72 v u_{x} u_{x x}-36 v u u_{x x x}-6 v_{x} u_{x}^{2}+\frac{32}{3} u^{3} v_{x}+96 v u^{2} u_{x} .
\end{aligned}
$$


As far as we know, Eqs. (10) and (11) have not been solved exactly. When $v=0$, Eqs. (10) and (11) are reduced to

$$
u_{t}=-u_{7 x}+49 u_{x} u_{x x x x}+14 u u_{5 x}+84 u_{x x} u_{x x x}-70 u_{x}^{3}-252 u u_{x} u_{x x}-56 u^{2} u_{x x x}+\frac{224}{3} u^{3} u_{x} \cdot
$$

In [2], Zhang and Tian obtained five new exact solutions including two hyperbolic function solutions, two trigonometric function solutions and a rational solution of Eq. (12) by using tanh-function method. The present paper is motivated by the desire to find new solutions of Eq. (12). Searching for exact solutions, especially new ones, of NLEEs is interesting because exact solutions can help us to gain more insight into some physical phenomena in many fields. Since the Korteweg-de Vries (KdV) equation was solved by Gardner, Green, Kruskal and Miura [3], many effective methods have been proposed for nonlinear PDEs. One of the existing methods is the so-called extended F-expansion method [4] proposed by Wang et al. Later, the extended F-expansion method [4] and its different improvements have received a wide range of applications, such as those in [5-12]. In this paper, the extended F-expansion method [4, 13] is employed to solve Eq. (12). As a result, many periodic wave solutions expressed by Jacobi elliptic functions are simultaneously obtained. These obtained periodic wave solutions can degenerate into solitary wave solutions and trigonometric function solutions in the limit cases of the modulus $m \rightarrow 1$ and $m \rightarrow 0$.

\section{Periodic Wave Solutions}

To begin with, we take the travelling wave transformation:

$u=u(\xi), \quad \xi=k x+\omega t$,

then Eq. (12) is transformed into an ordinary differential equation (ODE) of the form

$$
\begin{aligned}
\omega u^{\prime}= & -k^{7} u^{(7)}+49 k^{5} u^{\prime} u^{(4)}+14 k^{5} u u^{(5)}+84 k^{5} u^{\prime \prime} u^{\prime \prime \prime}-70 k^{3} u^{\prime 3}-252 k^{3} u u^{\prime} u^{\prime \prime} \\
& -56 k^{3} u^{2} u^{\prime \prime \prime}+\frac{224}{3} k u^{3} u^{\prime} .
\end{aligned}
$$

We next suppose that the solution of Eq. (14) can be expressed as

$$
u(\xi)=\sum_{i=-n}^{n} a_{i} F^{i}(\xi)
$$

where $a_{i}(i=0, \pm 1, \pm 2, \cdots, n)$ are constants to be determined, $n$ is an undetermined integer, while $F(\xi)$ satisfies Jacobi elliptic equation

$$
F^{\prime 2}(\xi)=P F^{4}(\xi)+Q F^{2}(\xi)+R
$$

and hence holds for $F(\xi)$ and $F^{\prime}(\xi)$

$$
\left\{\begin{array}{l}
F^{\prime \prime}(\xi)=2 P F^{3}(\xi)+Q F(\xi) \\
F^{\prime \prime \prime}(\xi)=\left(6 P F^{2}(\xi)+Q\right) F^{\prime}(\xi) \\
F^{(4)}(\xi)=24 P^{2} F^{5}(\xi)+20 P Q F^{3}(\xi)+\left(Q^{2}+12 P R\right) F(\xi) \\
\vdots
\end{array}\right.
$$

where $P, Q$ and $R$ are all parameters, the prime denotes $\mathrm{d} / \mathrm{d} \xi$. For Eq. (16), different Jacobi elliptic function solutions depending on parameters $P, Q$ and $R$ were listed in [13]. Noting that besides the 10 solutions [8] of Eq. (16) another 12 solutions were listed in [13] so that more solutions can be found for the considered nonlinear PDEs.

Balancing the highest order linear term $u^{(7)}$ with the highest order nonlinear term $u^{3} u^{\prime}$ in Eq. (14) yields $7+n=4 n+1$ which gives $n=2$. Thus, Eq. (15) becomes

$$
u=a_{0}+a_{1} F(\xi)+a_{2} F^{2}(\xi)+a_{-1} F^{-1}(\xi)+a_{-2} F^{-2}(\xi),
$$

With the help of Mathematica 4.0, we substitute Eq. (18) along with Eq. (17) into Eq. (14) and equate each coefficient of the same order power of $F^{\prime \rho}(\xi) F^{i}(\xi)(\rho=0,1 ; i=0, \pm 1, \pm 2, \cdots)$ to zero, a 
system of nonlinear algebraic equations for $a_{0}, a_{1}, a_{2}, a_{-1}, a_{-2}, P, Q, R, k$ and $\omega$ are obtained. Solving the system of nonlinear algebraic equations, we have three cases

$$
\begin{aligned}
& a_{0}=\frac{k^{2} Q}{2}, a_{1}=0, a_{2}=\frac{3 k^{2} P}{2}, \quad a_{-1}=0, a_{-2}=\frac{3 k^{2} R}{2}, \quad w=\frac{4}{3}\left(k^{7} Q^{3}-36 k^{7} P Q R\right), \\
& a_{0}=\frac{k^{2} Q}{2}, \quad a_{1}=0, \quad a_{2}=\frac{3 k^{2} P}{2}, \quad a_{-1}=0, \quad a_{-2}=0, \quad w=\frac{2}{3}\left(2 k^{7} Q^{3}-9 k^{7} P Q R\right), \\
& a_{0}=\frac{k^{2} Q}{2}, \quad a_{1}=0, \quad a_{2}=0, a_{-1}=0, a_{-2}=\frac{3 k^{2} R}{2}, \quad w=\frac{2}{3}\left(2 k^{7} Q^{3}-9 k^{7} P Q R\right),
\end{aligned}
$$

and hence obtain three formal solutions of Eq. (12) as follows

$$
\begin{aligned}
& u=\frac{k^{2} Q}{2}+\frac{3 k^{2} P}{2} F^{2}(\xi)+\frac{3 k^{2} R}{2} F^{-2}(\xi), \quad \xi=k x+\frac{4}{3}\left(k^{7} Q^{3}-36 k^{7} P Q R\right) t, \\
& u=\frac{k^{2} Q}{2}+\frac{3 k^{2} P}{2} F^{2}(\xi), \quad \xi=k x+\frac{2}{3}\left(2 k^{7} Q^{3}-9 k^{7} P Q R\right) t, \\
& u=\frac{k^{2} Q}{2}+\frac{3 k^{2} R}{2} F^{-2}(\xi), \quad \xi=k x+\frac{2}{3}\left(2 k^{7} Q^{3}-9 k^{7} P Q R\right) t,
\end{aligned}
$$

Selecting appropriate Jacobi elliptic functions $F(\xi)$ and different values of $P, Q$ and $R$ from Table 1 of [13] and using the formal solutions (20)-(22), we can obtain many periodic wave solutions expressed by Jacobi elliptic functions of Eq. (12). For example, if we select $F(\xi)=\operatorname{dn} \xi$, $P=-1, \quad Q=2-m^{2}$ and $R=m^{2}-1$, then Eq. (21) gives

$$
u=\frac{k^{2}\left(2-m^{2}\right)}{2}-\frac{3 k^{2}}{2} \operatorname{dn}^{2}(\xi)
$$

where

$$
\xi=k x+\frac{2}{3}\left[2 k^{7}\left(2-m^{2}\right)^{3}+9 k^{7}\left(2-m^{2}\right)\left(m^{2}-1\right)\right] t .
$$

In Fig. 1, a spatial structure of solution (23) with $m=0.5$ and $k=1$ is shown. Fig. 2 describes the corresponding dynamical evolutions. In Figs. 2 and 3, another spatial structure of solution (23) with $m=0.01$ and $k=1$ and the corresponding dynamical evolutions are described, respectively. It is easy to see from Figs. 1-4 that solution (30) is a double periodic wave solution.

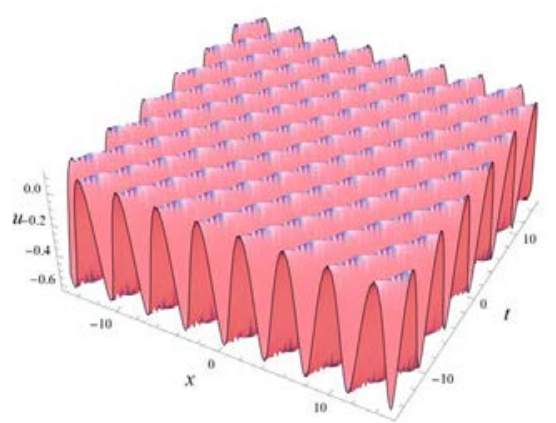

Fig. 1. Spatial structure of double periodic wave solution (23).

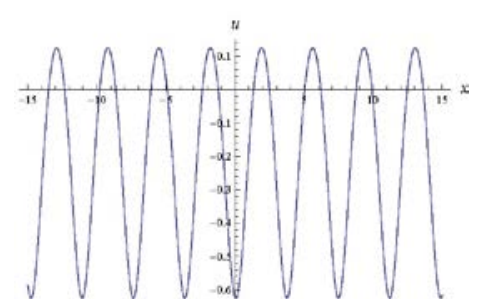

$t=-5$

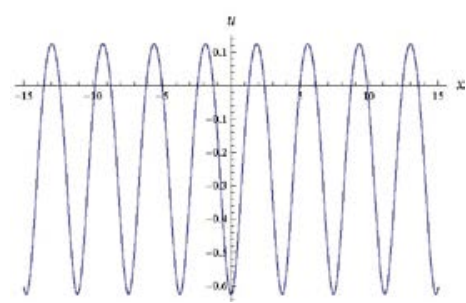

$t=0$

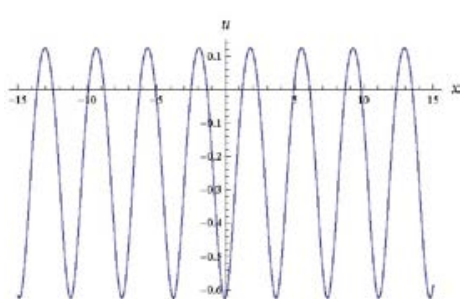

$t=5$

Fig. 2. Dynamical evolutions of double periodic wave solution (23). 
In the limit case $m \rightarrow 1$, solution (30) degenerate into a solitary wave solution $u=\frac{k^{2}}{2}-\frac{3 k^{2}}{2} \operatorname{sech}^{2}(\xi), \quad \xi=k x+\frac{4 k^{7}}{3} t$,

which is displayed in Fig. 5. In Fig. 6, the corresponding dynamical evolutions are shown.

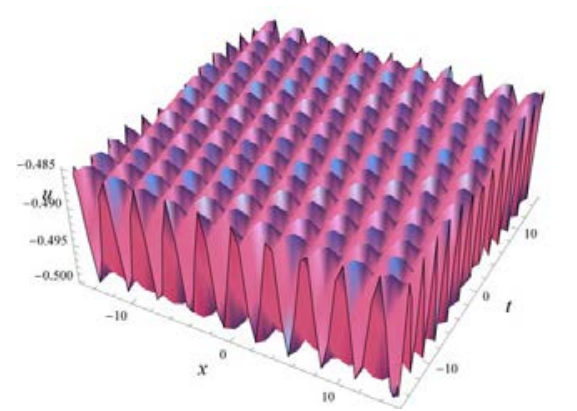

Fig. 3. Spatial structure of double periodic wave solution (25).
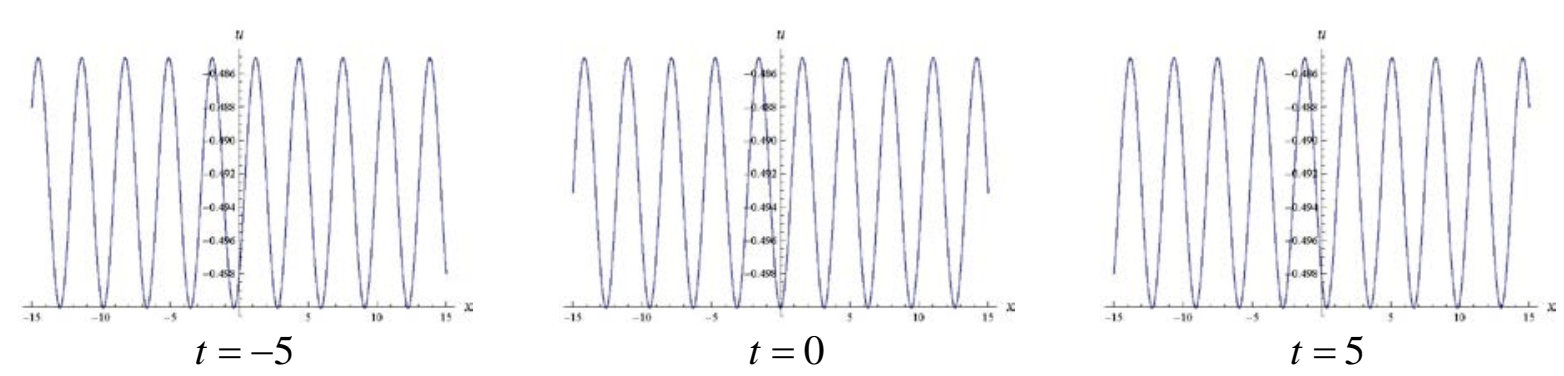

Fig. 4. Dynamical evolutions of double periodic wave solution (25).

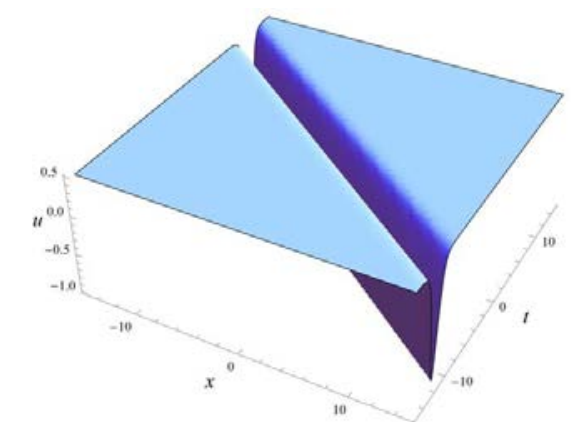

Fig. 5. Spatial structure of solitary wave solution (25).
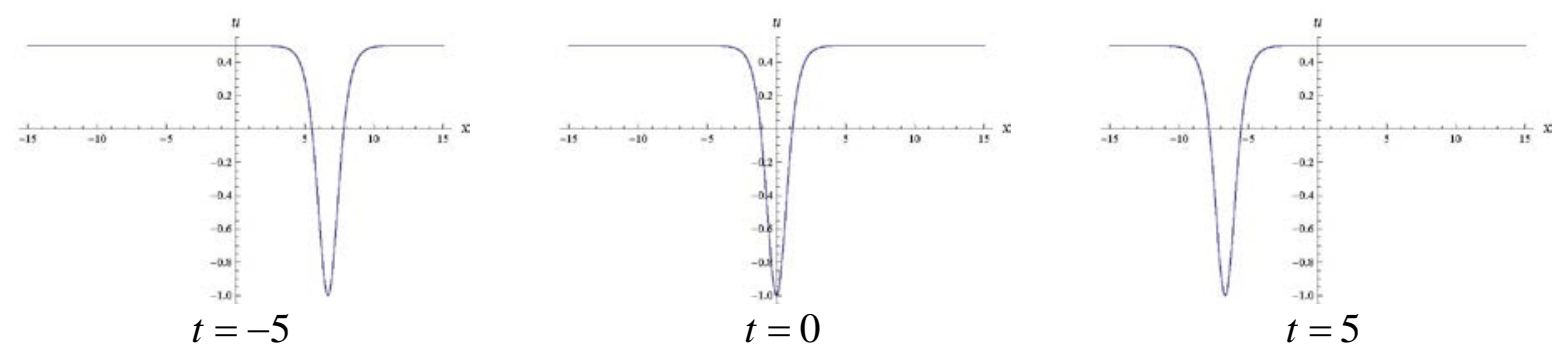

Fig. 6. Dynamical evolutions of solitary wave solution (25).

\section{Conclusion}

In summary, with the aid of computerized symbolic computation we have successfully obtained many periodic wave solutions expressed by Jacobi elliptic functions of one reduced equation (12) of the MNW hierarchy (2) by using the extended F-expansion method [4, 13]. To the best of our knowledge, these obtained periodic wave solutions are new. Three spatial structures with dynamical evolutions of the obtained solution (23) are shown by means of computer simulation. The obtained 
periodic wave solutions expressed by Jacobi elliptic function solutions can degenerate into solitary wave solutions and trigonometric function solutions in the limit cases. This paper shows that the extended F-expansion method [4, 13] combined with computerized symbolic computation can provide a preferred mathematical tool for finding new periodic wave solutions of some other new nonlinear PDEs.

\section{Acknowledgement}

This work was supported by the Natural Science Foundation of China (11547005), the PhD Start-up Fund of Liaoning Province of China (20141137) and the Liaoning BaiQianWan Talents Program (2013921055).

\section{References}

[1] Xiaoying Shan, Junyi Zhu. The Mikhauilov-Novikov-Wang hierarchy and its Hamiltonian structures. Acta Physica Polonica B, 43 (2012) 1953-1963.

[2] Sheng Zhang, Chi Tian. A new mathematical model and its solvability test on computer. Advances in Engineering Research, 97 (2016) 375-379.

[3] Clifford Garder, John Greene, Martin Kruskal, Robert Miura. Method for solving the Korteweg-de Vries equation. Physical Review Letters, 19 (1967) 1095-1097.

[4] Yubing Zhou, Minglian Wang, Yueming Wang. Periodic wave solutions to a coupled KdV equation with variable coefficients. Physics Letters A, 308 (2003) 31-36.

[5] Mingliang Wang, Yubin Zhou. The periodic wave solutions for the Klein-Gordon-Schrodinger equations. Physics Letters A, 318 (2003) 84-92.

[6] Sheng Zhang. The periodic wave solutions for the (2+1)-dimensional KonopelchenkoDubrovsky equations. Chaos Solitons \& Fractals, 30 (2006) 1213-1220.

[7] Sheng Zhang. New exact solutions of the KdV-Burgers-Kuramoto equation. Physics Letters A, 358 (2006) 414-420.

[8] Sheng Zhang, Tiecheng Xia. A generalized F-expansion method and new exact solutions of Konopelchenko-Dubrovsky equations. Applied Mathematics and Computation, 183 (2006) 11901200.

[9] Sheng Zhang. The periodic wave solutions for the (2+1)-dimensional dispersive long water equations. Chaos Solitons \& Fractals, 32 (2007) 847-854.

[10] Sheng Zhang. Further improved F-expansion method and new exact solutions of KadomstevPetviashvili equation. Chaos Solitons \& Fractals, 32 (2007)1375-1383.

[11] Sheng Zhang, Tiecheng Xia. A generalized F-expansion method with symbolic computation exactly solving Broer-Kaup equations. Applied Mathematics and Computation, 189 (2007) 836843.

[12] Sheng Zhang, Tiecheng Xia. An improved generalized F-expansion method and its application to the (2+1)-dimensional KdV equations. Communications in Nonlinear Science and Numerical Simulation, 13 (2008) 1294-1301.

[13] Sheng Zhang, Yingna Sun, Jinmei Ba, Ling Dong. Explicit and exact solutions with multiple arbitrary analytic functions of Jimbo-Miwa equation. Applications and Applied Mathematics: An International Journal, 4 (2009) 279-289 